$$
\text { "tmcs-csabay" — 2011/5/13 — 21:42 — page } 27 \text { — }
$$

\title{
Combinatorics teaching experiment
}

\author{
KÁroly Csabay and JózSef Daragó
}

Abstract. Teaching combinatorics has got its conventional method. One has to see: the combinatorical formations won't be follow each other by a heuristic way. The formulas kept by pupils seem to come from "deus ex machina". We try to offer now an alternative way to approach combinatorical concepts from a nontraditional direction and point of view.

Key words and phrases: combinatorics, didactic, generalized Pascal-triangle.

ZDM Subject Classification: D30, D40, K20.

The traditional path

The traditional path in which teachers act when they teach elementary combinatorics is:

Schema 0:

- Separating cases in which repetitions are allowed, and, when they aren't

- Fixing that combinatorical configurations without repetitions are "easy", with them they, however, are "difficult"

- Starting with the combinatorical configurations without repetitions teachers used to explain

permutations (it's easy indeed, pupils learn the concept of factorials quickly) variations (teachers used to cut off the tail of the factorial product when its sort order is descending; so they get a product which gives the number of the variations - easy again)

Copyright (C) 2011 by University of Debrecen 
combinations (here they used to exploit that variations are permutations of the combinations, so the number of the combinations can be obtained by a simple division - also easy) - and as a bypass pupils become familiar with the binomial coefficients $\left(\begin{array}{l}n \\ k\end{array}\right)$

- Now it will be continued with repetitions

permutations with repetitions will be taught as a product of certain binomial coefficients (introduced above)

variations with repetitions are quite different from the queue, teachers do not make attentive, in spite

combinations: the teacher is showing the "trick" (the concept of the $k-1$ phantom pupils when it is built to obtain $k$ pieces of chocolates)

The didactical method, implemented under the name "Schema 0 " is a customized, traditional way. Let's take a look at the following formulas!

$$
\frac{n !}{n_{1} ! n_{2} ! \ldots n_{r} !} \text { and } n^{k} \text { and }\left(\begin{array}{c}
n+k-1 \\
k
\end{array}\right)
$$

There's no relationship among them according formal point of view, either. Now we try to offer a parallel way, which, we believe, will help students in understanding of the origin of the formulas.

\section{Alternative suggestions}

We would like to give now some basic rules. They are:

\section{Our rules}

Rule 1: Don't begin with permutations! (I. e. you need to introduce them only at the finishing stage.)

Rule 2: Interpret ideas as "stock" and "pattern". Write these words capitalized: Stock, Pattern. Have element number of the Stock be signed always by $n$, and, the length of the Pattern always by $k$.

Rule 3: Start always with the simplest examples!

Rule 3/a: Remember, the simplest case mustn't be the case of zero! You are a mathematician, you like zero because of its comfortable behavior. Children, however, feel zero to be strange. Begin at the number one!

Rule 4: Feel free to rely on the fact how the formulas inherit their values! 


$$
\text { "tmcs-csabay" — 2011/5/13 — 21:42 — page } 29 \text { — \#3 }
$$

\section{So now let's start with!}

We introduce now the following concepts: Stock and Pattern. The Stock could be imagined as a bag in which one can find pearls, marbles, colors, chocolates, cards, and so on. The Pattern is a certain string built of the elements of the Stock. For example, a string of pearls may be called a Pattern. We make clear that we'll distinguish cases in which the same element can be reused in the Pattern and cases when it mustn't. On the other hand we make a difference between cases when the order of the Pattern is relevant, and, when it isn't.

\section{Examples}

Now we have to give some examples to the concepts introduced above.

Example 1. Let us dice! Suppose, we play with two dice. Our Stock contains the numbers on the die: $1,2,3,4,5,6(n=6)$. The length of the Pattern is now two, because there are two dice $(k=2)$. Question for the possibility whether a number could be cast twice. Of course! Now, each element can be repeated. Consider that our two dice are totally identical. After casting there's no method to distinguish the case $4-5$ and the case $5-4$. So our Pattern now contains two positions but the sequence of the positions is irrelevant.

EXAmple 2. Let us dice just as above! $(n=6, k=2$.) The difference is, that the first die is white having black spots on it, the other, by contrast, is black, having white spots on it. After casting there's a clear method to distinguish the case 4-5 and the case 5-4, namely one case: white 4 , black 5 ; another case: white 5 , black 4 . So our Pattern now contains two positions again but their sequence is relevant.

EXAMPLE 3. Let be our Stock the colors, and we try to collect flags consisting of three stripes. For instance our colors could be: red, green, blue, white, black, yellow. So we've obtained a Stock of six elements $(n=6)$. Here we see a Pattern of three positions $(k=3)$. Is the sequence of the colors relevant? Of course it is! An example can be the Hungarian and Bulgarian flag. The yonder is red-whitegreen, the next is white-green-red.

EXAMPle 4. Imagine a drag in the lottery. Consider that this game consists of 45 numbers $(n=45)$ and six numbers will be drown $(k=6)$. Is it possible that 
a certain number will be drown twice? No, it is impossible. Does the sequence of the six drown number play any role? No, it doesn't.

Pupils will - by such or several similar examples - become familiar with the questions about Stock, Pattern, sequence, and, repetition. We have now reached the level that we may set the following task:

\section{Expressing tasks (order counts)}

We have got (a sufficient amount of) marbles of $\mathrm{n}$ several colors, we intend to build them to a string of $k$-length. Repetitions are allowed, the several sequences count in different. For example we have got red $(R)$, green $(G)$ and blue $(B)$ marbles $(n=3)$ and we have to set strings of three marbles $(k=3)$. And - by a parallel - we also target the formations: strings without a repetition.

Solutions in the first case are:

RRR, RRG, RRB, RGR, RGG, RGB, RBR, RBG, RBB,

GRR, GRG, GRB, GGR, GGG, GGB, GBR, GBG, GBB,

BRR, BRG, BRB, BGR, BGG, BGB, BBR, BBG, BBB.

Solutions in the second case are:

$$
\begin{array}{ll}
\text { RGB, } & \text { RBG, } \\
\text { GRB, } & \text { GBR, } \\
\text { BRG, } & \text { BGR. }
\end{array}
$$

Here we see 27 and 6 solutions, respectively, and we wonder if we could tell this number for all $n$ and $k$.

\section{Steps to solution}

Let's focus to the first problem (repetitions allowed) and draw a table:

\begin{tabular}{|c|c|c|c|c|}
\cline { 3 - 5 } \multicolumn{2}{c|}{} & \multicolumn{3}{c|}{ Edge for $k$} \\
\cline { 2 - 5 } \multicolumn{2}{c|}{} & 1 & 2 & 3 \\
\hline \multirow{2}{*}{$\begin{array}{c}\text { Edge } \\
\text { for } \\
n\end{array}$} & 1 & $n=1$ and $k=1$ & $n=1$ and $k=2$ & $n=1$ and $k=3$ \\
\cline { 2 - 5 } & 2 & $n=2$ and $k=1$ & $n=2$ and $k=2$ & $n=2$ and $k=3$ \\
\cline { 2 - 5 } & 3 & $n=3$ and $k=1$ & $n=3$ and $k=2$ & $n=3$ and $k=3$ \\
\hline
\end{tabular}


Usually the simplest case is when both $n$ and $k$ equal to 1 . We consult this case at the beginning. When both of the parameters equal to one then we can build only one color marble string: the lonely marble will represent the one possible string. So our table looks now like:

\begin{tabular}{|l|l|l|l|}
\cline { 2 - 4 } \multicolumn{1}{c|}{} & 1 & 2 & 3 \\
\hline 1 & 1 & & \\
\hline 2 & & & \\
\hline 3 & & & \\
\hline
\end{tabular}

Next we walk through the edges. Fix $k$ in 1 , and investigate how the first column of our table depends on $n$. In other words: how many strings can be built when we have got $n$ several marbles and the length of the string must be one. We can build $n$ such strings. Each marble can have a role in the Pattern - and we got ready. Let's proceed filling our table!

\begin{tabular}{|l|l|l|l|}
\cline { 2 - 4 } \multicolumn{1}{c|}{} & 1 & 2 & 3 \\
\hline 1 & 1 & & \\
\hline 2 & 2 & & \\
\hline 3 & 3 & & \\
\hline
\end{tabular}

Now we fix $n=1$. When $n=1$ we examine how the values depend on $k$. There is one color only - let it be e.g. the red color! How many $k$-length strings can be built from red marbles? The answer is always 1, independently of $k$. Our table now looks like:

\begin{tabular}{|l|l|l|l|}
\cline { 2 - 4 } \multicolumn{1}{c|}{} & 1 & 2 & 3 \\
\hline 1 & 1 & 1 & 1 \\
\hline 2 & 2 & & \\
\hline 3 & 3 & & \\
\hline
\end{tabular}

Our aim is to fill out the whole table. How do we fill the table? From left to right and from top to bottom. When we try to figure out the value of the next empty cell we hope to get help from its left, upper-left, or, upper neighbor. In this case, it is sufficient to rely on the left-hand neighbor. Let's consider, we have to fill the cell $(n, k)$. Its left neighbor is $(n, k-1)$. When we know the value of $(n, k-1)$, how do we compute the value of $(n, k)$ ? Again, when we know the value 
of a shorter Pattern, how can we gain the value of the longer Pattern? Easy: each shorter Pattern can be lengthened by any of the $n$ marbles of the Stock. So:

$$
\text { value of }(n, k)=\text { value of }(n, k-1) \text { multiplied by } n
$$

For sake of a more mathematic-like writing we introduce the typo as:

$$
V(n, k)=V(n, k-1) \cdot n
$$

Teachers can use here the capabilities of Excel. Students are probably familiar with Excel, they know its column naming scheme: A, B, C,..., and its row identification system: $1,2,3, \ldots$, as well. Filling the cell C3 you have to write the expression: $=\mathrm{B} 3 * \$ \mathrm{~A} 3$

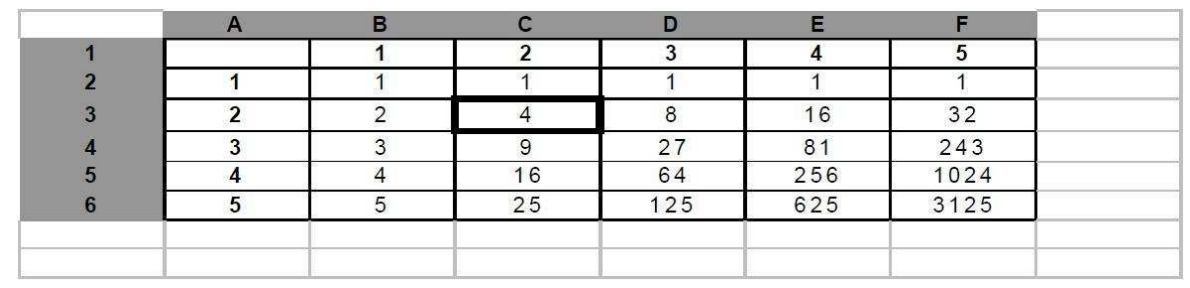

The meaning of the Excel-syntax in this case is: to get the value of C3 its left neighbor (B3) should be multiplied by the corresponding $n$ (A3). The goal why we use the $\$$ symbol is making our expression able to have been copied. B3, addressed from C3 tells Excel to think of the left-hand neighbor cell - this addressing mode is relative. The cell-reference $\$ A 3$ tells Excel not to move the column "A" when the expression will have been copied. So the expression B3*\$A3, when having been copied into cell D3 becomes $\mathrm{C} 3 * \$ \mathrm{~A} 3$, while the variant B3*A3 would have been changed to $\mathrm{C} 3{ }^{*} \mathrm{~B} 3$ - improperly.

So we taught variations with repetitions without speaking about them.

The logics for the second case (repetitions disallowed) are similar. The left upper corner and the edges will be filled identically as at the first case. Now, when we look at the smaller Pattern and we try to attach to it a new marble, we can agree that in the smaller Pattern can have a role only a subset of the Stock: namely, the $n^{\text {th }}$ marble must not take a part in the smaller Pattern. So we have gained:

$$
\text { value of }(n, k)=\text { value of }(n-1, k-1) \text { multiplied by } n
$$

For sake of a more mathematic-like writing we introduce the typo as:

$$
W(n, k)=W(n-1, k-1) \cdot n
$$


The appropriate Excel formula to compute the value of the cell C3 is now: $=\mathrm{B} 2 * \$ \mathrm{~A} 3$

\section{Let's turn to combinations!}

By the first we have to decide what will be the case to start with. Either the case with repetitions or the case without repetition. Let's have the following Stock and Pattern: the Stock will be the community of the $n$ children in the classroom, the Pattern will be a $k$-elements set of chocolates. We investigate the question how many solutions there are to distribute $k$ pieces of chocolates among $n$ children.

The situation when we do not allow repetitions is meaning in this context that a certain child may get at most one piece of chocolate. Well, now we put the task:

Putting the task: $k$ chocolates to $n$ children (repetition allowed)

How many solutions are there when we intend to distribute $k$ pieces of chocolates among $n$ children, and a child can get any number of chocolates?

Let's investigate the case $n=k=1$. It is plausible to see that the number of the solutions is 1 . So we have now a table as:

\begin{tabular}{|l|l|l|l|}
\cline { 2 - 4 } \multicolumn{1}{c|}{} & 1 & 2 & 3 \\
\hline 1 & 1 & & \\
\hline 2 & & & \\
\hline 3 & & & \\
\hline
\end{tabular}

When we fix $n$ in 1 then the number of the solutions are always 1 independently of the actual value of $k$. Our table looks now like:

\begin{tabular}{|l|l|l|l|}
\cline { 2 - 4 } \multicolumn{1}{c|}{} & 1 & 2 & 3 \\
\hline 1 & 1 & 1 & 1 \\
\hline 2 & & & \\
\hline 3 & & & \\
\hline
\end{tabular}

We walk along the edge $k=1$ : there's only one piece of chocolate and children are more than one. The number of the distributions can be obtained: it equals to $n$. We have got so the table again: 


$$
\text { "tmcs-csabay" — 2011/5/13 — 21:42 — page } 34-\# 8
$$

\begin{tabular}{|l|l|l|l|}
\cline { 2 - 4 } \multicolumn{1}{c|}{} & 1 & 2 & 3 \\
\hline 1 & 1 & 1 & 1 \\
\hline 2 & 2 & & \\
\hline 3 & 3 & & \\
\hline
\end{tabular}

How do we continue? Having solutions for either a shorter Stock $(n-1$ children) or a shorter Pattern ( $k-1$ chocolates) we start to think: how could be calculated the number of the distributions of $k$ chocolates among $n$ children? Fix the point of view onto the $n^{\text {th }}$ child! There are only two possibilities: either he/she gets a chocolate or he/she does not get any.

- So when the $n^{\text {th }}$ child is getting a piece of chocolate then the remainder $k-1$ pieces should be distributed among $\mathrm{n}$ children (remember! the $n^{\text {th }}$ child also can get another chocolates!).

- And when the $n^{\text {th }}$ child isn't getting a chocolate then the $k$ pieces of chocolates should be distributed among $n-1$ children.

Because we discussed two distinct cases, the numbers of solutions may be added:

$$
\text { value of }(n, k)=\text { value of }(n, k-1) \text { added to value of }(n-1, k)
$$

For sake of a more mathematic-like writing we introduce the typo as:

$$
C(n, k)=C(n, k-1)+C(n-1, k)
$$

Without drawing a worksheet again we can tell simply the appropriate Excel formula to compute the value of the cell $\mathrm{C} 3$ is: $=\mathrm{B} 3+\mathrm{C} 2$

The formula can be copied to the whole pane we intend to use. (Note that in this case you needn't apply the $\$$ sign! Neighbor cells are addressed in a relative manner.)

Putting the task: $k$ chocolates to $n$ children (no repetitions)

Now we have discussed completely the case in which repetitions are allowed. It is the moment when the teacher can turn to the case when repetitions aren't allowed. There are $n$ children and $k$ pieces of chocolates again. Our task is to figure out the number of the possibilities how many ways there are, having chocolates allocated to children when each child may get only one chocolate at most.

Let's start with the upper left corner as usual! In the case $n=k=1$ it is easy to see that the number of the possibilities is 1 . So we have now a table as: 


$$
\text { "tmcs-csabay" — 2011/5/13 — 21:42 — page } 35 \text { — \#9 }
$$

\begin{tabular}{|l|l|l|l|}
\cline { 2 - 4 } \multicolumn{1}{c|}{} & 1 & 2 & 3 \\
\hline 1 & 1 & & \\
\hline 2 & & & \\
\hline 3 & & & \\
\hline
\end{tabular}

When we fix $n$ in 1 and $k>1$ then we see that more than one chocolates cannot be given to one child. Our table looks now like:

\begin{tabular}{|l|l|l|l|}
\cline { 2 - 4 } \multicolumn{1}{c|}{} & 1 & 2 & 3 \\
\hline 1 & 1 & 0 & 0 \\
\hline 2 & & & \\
\hline 3 & & & \\
\hline
\end{tabular}

When we deal with the edge $k=1$ we get the table (the clue is exactly the same as it was formerly):

\begin{tabular}{|l|l|l|l|}
\cline { 2 - 4 } \multicolumn{1}{c|}{} & 1 & 2 & 3 \\
\hline 1 & 1 & 0 & 0 \\
\hline 2 & 2 & & \\
\hline 3 & 3 & & \\
\hline
\end{tabular}

The line continues to the filling of the remainder cells. We focus to the $n^{\text {th }}$ child again. And, again, there are now two distinct cases: the $n^{\text {th }}$ child does get a chocolate, or, the $n^{\text {th }}$ child does not get any chocolates.

- When the $n^{\text {th }}$ child does get a chocolate then the remainder $k-1$ pieces of chocolates can be allocated among the remainder $n-1$ children.

- When the $n^{\text {th }}$ child does not get any chocolates then all the $k$ pieces of chocolates can be allocated among the remainder $n-1$ children.

Owing to our considerations we can say that:

$$
\text { value of }(n, k)=\text { value of }(n-1, k) \text { added to value of }(n-1, k-1)
$$

For sake of a more mathematic-like writing we introduce the typo as:

$$
K(n, k)=K(n-1, k)+K(n-1, k-1)
$$

The appropriate Excel formula to compute the value of the cell $\mathrm{C} 3$ is: $=\mathrm{B} 2+\mathrm{C} 2$
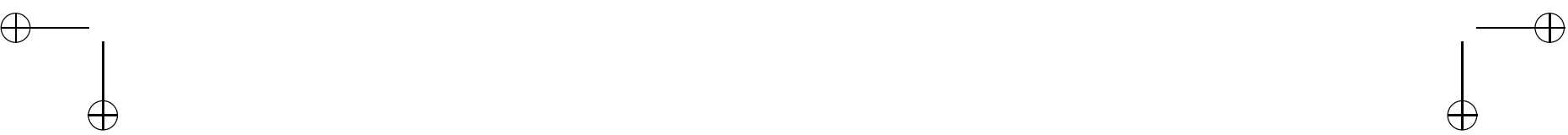


\section{A comprehensive overview}

We have got four tables, table V, W, C, and, K. Let's have summarized in a table how we start to fill cells in them:

\section{Table 1}

\begin{tabular}{|c|c|c|c|}
\hline The table & Left upper corner & Edge $n=1$ & Edge $k=1$ \\
\hline $\mathrm{V}$ & 1 & 1 & $n$ \\
\hline $\mathrm{W}$ & 1 & 0 & $n$ \\
\hline $\mathrm{C}$ & 1 & 1 & $n$ \\
\hline $\mathrm{K}$ & 1 & 0 & $n$ \\
\hline
\end{tabular}

\section{Pictures to illuminate}

Now we draw four pictures by which we intend to illustrate on which neighbors the value of a cell depend when we calculate it from the previous values.

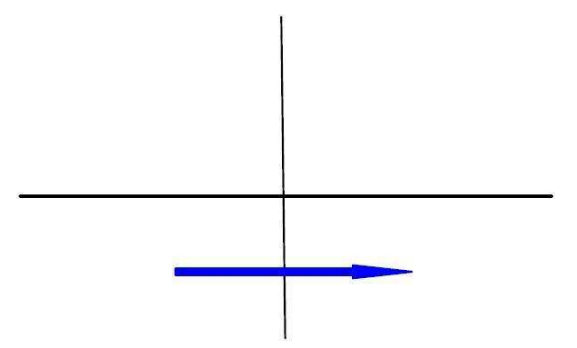

Figure 1. Calculation in the V-table: it depends only on the lefthand neighbor

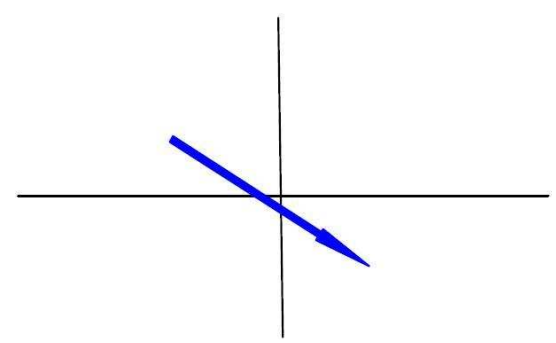

Figure 2. Calculation in the W-table: it depends only on the leftupper neighbor 


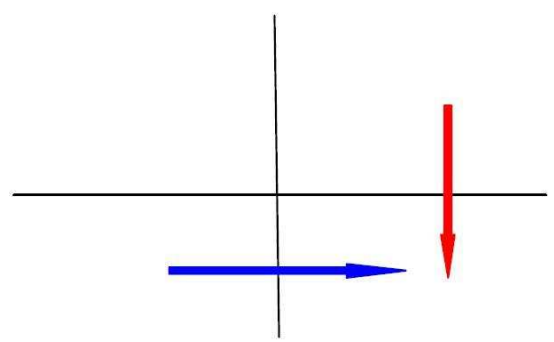

Figure 3. Calculation in the C-table: it depends on the left and the upper neighbor

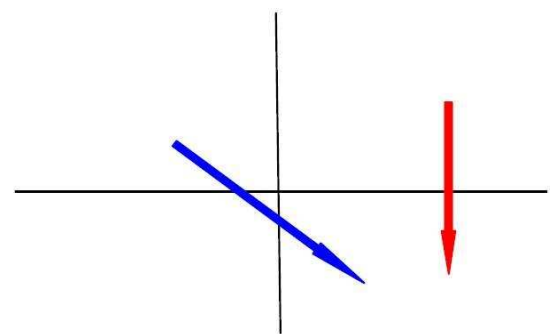

Figure 4. Calculation in the K-table: it depends on the left-upper and the upper

What do we do when we calculate these cells? We relay onto these neighborhoods: left, left-upper, and, upper.

\section{Generalized Pascal-triangle}

The notation system from now on will be a loan from Paul Barry. [1] Mr. Barry calls such matrices as "Pascal- $(x, y, z)$-arrays", where the coefficients $x, y$, and, $z$ refer to the left, left-upper, and, upper neighbor cells, respectively. As Mr. Barry has written (by e-mail): "Pascal- $(x, y, z)$-array" is one of my own invention, and it describes the square array $T(n, 0)=T(0, k)=1, T(n, k)=$ $x * T(n, k-1)+y * T(n-1, k-1)+z * T(n-1, k)$.

In the Barry-interpretation there is a limitation: only constant values can be given to $x, y, z$. Now we have to widen this allowance: these parameters might have also such values as $n$ or $k$. In this case we can summarize our state in a table as:

Now let us combine our tables Table 1 and Table 2 . 


$$
\text { "tmcs-csabay" — 2011/5/13 — 21:42 — page } 38 \text { — \#12 }
$$

Table 2

\begin{tabular}{|c|c|c|c|}
\hline The table & $\begin{array}{c}\text { Coefficient } \\
\text { for the left } \\
\text { neighbor }(x)\end{array}$ & $\begin{array}{c}\text { Coefficient } \\
\text { for the left-upper } \\
\text { neighbor }(y)\end{array}$ & $\begin{array}{c}\text { Coefficient } \\
\text { for the upper } \\
\text { neighbor }(z)\end{array}$ \\
\hline $\mathrm{V}$ & $n$ & 0 & 0 \\
\hline $\mathrm{W}$ & 0 & $n$ & 0 \\
\hline $\mathrm{C}$ & 1 & 0 & 1 \\
\hline $\mathrm{K}$ & 0 & 1 & 1 \\
\hline
\end{tabular}

Compact tableaus

Table 1-2

\begin{tabular}{|c|c|c|c|c|c|c|}
\hline & $n=k=1$ & $n=1$ & $k=1$ & $x$ & $y$ & $z$ \\
\hline $\mathrm{V}$ & 1 & 1 & $n$ & $n$ & 0 & 0 \\
\hline $\mathrm{W}$ & 1 & 0 & $n$ & 0 & $n$ & 0 \\
\hline $\mathrm{C}$ & 1 & 1 & $n$ & 1 & 0 & 1 \\
\hline $\mathrm{K}$ & 1 & 0 & $n$ & 0 & 1 & 1 \\
\hline
\end{tabular}

This table can be considered as an effective tool. The lines of this table could be continued e.g. as:

For a small outlook

Table 1-2+

\begin{tabular}{|c|c|c|c|c|c|c|}
\hline & $n=k=1$ & $n=1$ & $k=1$ & $x$ & $y$ & $z$ \\
\hline $\mathrm{V}$ & 1 & 1 & $n$ & $n$ & 0 & 0 \\
\hline $\mathrm{W}$ & 1 & 0 & $n$ & 0 & $n$ & 0 \\
\hline $\mathrm{C}$ & 1 & 1 & $n$ & 1 & 0 & 1 \\
\hline $\mathrm{K}$ & 1 & 0 & $n$ & 0 & 1 & 1 \\
\hline Stirl2 & 1 & 0 & 1 & 0 & 1 & $k$ \\
\hline InvB & 1 & 0 & 0 & 0 & 1 & -1 \\
\hline Euler & 1 & 1 & 1 & $n$ & 0 & $k$ \\
\hline St?? & 1 & 1 & 1 & 1 & -2 & 1 \\
\hline Del & 1 & 1 & 1 & 1 & 1 & 1 \\
\hline
\end{tabular}

The exemplars above - beyond V, W, C, and, K - are: 


$$
\text { "tmcs-csabay" — 2011/5/13 — 21:42 — page } 39 \text { - \#13 }
$$

Stirl2 Stirling numbers of the second kind

InvB Inverted binomial triangle

Euler Elements of Euler's triangle

St?? A bit strange numbers which make remember Steinbach numbers

Del Delannoy numbers

There are also plausible generations for Bell-, Leibniz-, Narayana-, and, Deleham numbers, and, for several Riordan-arrays, as well. [2]-[6]

\section{Nomenclature}

By a return to our original line, we could investigate "Table 1" again. It is remarkable that lines $\mathrm{V}$ and $\mathrm{C}$ are identical. On the other hand lines $\mathrm{W}$ and $\mathrm{K}$ are identical again. What was the relationship between lines V and C? They were the "with repetition" cases. And, what was the relationship between lines W and K? They were the "without repetition" cases. Let's investigate now "Table 2". The lines $\mathrm{V}$ and $\mathrm{C}$ show the dependency on the left neighbor. The lines $\mathrm{W}$ and $\mathrm{K}$ show the dependency on the left-upper neighbor. The lines $\mathrm{W}$ and $\mathrm{K}$ - in addition show the dependency on the upper neighbor.

The calculated sheets we have gained by obeying the upper instructions represent numbers that we call

variations (cases $\mathrm{V}$ and $\mathrm{W}$ )

alternatively

combinations (cases $\mathrm{C}$ and $\mathrm{K}$ )

Evidently we say

variation with repetition in the case $\mathrm{V}$

and we say

combination with repetition in the case $\mathrm{C}$

obviously we say

variation without repetition in the case $\mathrm{W}$

and we say

combination without repetition in the case $\mathrm{K}$

Well, we have reached now a level where we can originate these ideas from a common root. One might hope that this approach will please learners. At this point teachers can define the formula $K(n, k)$ as $\left(\begin{array}{l}n \\ k\end{array}\right)$ and they express its name: binomial coefficient. It is an attractive task to proof the equation $C(n, k)=$ 
$\left(\begin{array}{c}n+k-1 \\ k\end{array}\right)$. It is significantly easier proofing, by the way, the statement $V(n, k)=$ $n^{k}$. Where do we start now with $W(n, k)$ ?

\section{Upcoming permutations}

An idea helping to solve this problem, can be a restricted investigation; at the first we look at $W(n, n)$. Remember that in our first example was $n=k=3$. (There were three colors and three positions for marbles.) The result was 6 . So we know now that $W(3,3)=6$. $W(4,4)$ comes from $W(3,3)$ multiplied by 4 . And then $W(5,5)$ comes from $W(4,4)$ multiplied by 5 . And so on. $W(n, n)$ comes from $W(n-1, n-1)$ multiplied by $n$. A sequence having such a regularity can be easily accepted by learners, and teachers can define - relaying on it - factorials. Here also can be defined its symbol: $n$ !

So we can see as permutations appear as a special case of variations.

People like regular things. When you try to supply "Table 1-2" by an additional hypothetical row as

\begin{tabular}{|c|c|c|c|c|c|c|}
\hline & $n=k=1$ & $n=1$ & $k=1$ & $x$ & $y$ & $z$ \\
\hline $\mathrm{P}$ & 1 & 1 & $n$ & 0 & 0 & $n$ \\
\hline
\end{tabular}

so you can study how columns report factorials (each column reports the same).

Back to the variations

Before continuing, teachers have to absolve an unpleasant task. They need to introduce the factorial of the zero. The most comfortable way for this could be the "ex cathedra" method: the teacher is expressing explicitly that $0 !=1$. Teachers can tell also the absence of a negative factorial - when so, we count the formula as it expressed a zero. These conventions will help at the next step as we'll express the number of the variations by factorials. By return to the question about $W(n, k)$ now we have already got the tools. Now we are allowed to say:

$$
W(n, k)=\frac{n !}{(n-k) !}
$$

Making the proof we return to the roots: namely to the basic values $W(1,1)$; $W(n, 1)$, and, $W(1, k)$. (In these formulas $(n, 1)$ is a shorthand for $(n, 1) \& n>1$; literally: $(1, k)$ is for $(1, k) \& k>1$.) So: 


$$
W(1,1)=\frac{1 !}{0 !}=1 \quad W(n, 1)=\frac{n !}{(n-1) !}=n \quad W(1, k)=\frac{1 !}{\langle\text { negative } !}=0
$$

The recursion can be controlled as follows:

$$
W(n, k)=\frac{n !}{(n-k) !}=\frac{n(n-1) !}{(n-1-(k-1)) !}=n \cdot W(n-1, k-1)
$$

The new scheme

Schema 1:

- Defining ideas as Stock and Pattern (having $n$ and $k$ element, respectively)

- Separating cases in which repetitions are allowed, and, when they aren't

- Separating cases in which the order of the Pattern is relevant, and, when it isn't

- Fixing that a Pattern bigger than the Stock $(k>n)$ cannot be filled without repetitions (so in these cases the number of the solutions will be always 0 )

- Fixing that when repetitions are allowed then a smaller Pattern can hold the same Stock, when, by contrast, repetition are disallowed then a smaller Pattern cannot hold the same Stock; in this case the Stock has to be decremented

- Fixing that when order of the Pattern is relevant then the step from the smaller Pattern to the bigger Pattern consist of a multiplication by $n$

- Fixing that when order of the Pattern is irrelevant then the step from the smaller Pattern to the bigger Pattern consist of an addition to the "the same Pattern with the smaller Sock" formula

- So we deal with two oppositions: 


$$
\text { "tmcs-csabay" — 2011/5/13 — 21:42 — page } 42 \text { — \#16 }
$$

\section{Repetition allowed}

A smaller Pattern can hold the same Stock

Values inherit from the left cell

The Pattern can be greater than the Stock

In the case $n=1(\& k>1)$ the cells of the table edge contains of 1 -s

\section{Order is relevant}

The step from a smaller Pattern is a multiplication by $n$

The upper neighbor does not take a The upper neighbor does take a role role

\section{Independent features}

The table shows in the case $n=k=1$ always 1

The table shows in the case $k=1$ always $n$

\section{Mathematical background}

In general, we speak of a two-dimensional function as:

$$
A(n, k)=\left\{\begin{aligned}
& a(n, k) \text { if } n=1 \& k=1 \\
& b(n, k) \text { if } n=1 \& k>1 \\
& c(n, k) \text { if } k=1 \& n>1 \\
& x(n, k) A(n, k-1)+y(n, k) A(n-1, k-1)+ \\
&+z(n, k) A(n-1, k)+d(n, k) \text { otherwise }
\end{aligned}\right.
$$


Readers can investigate an implementations of a simplified example of this function on the practicing page here: http://konyvtar.mezobereny.hu/csabay/ Debrecen/teszt/Racs/Ank.php

Among certain circumstances these grids will be symmetric, and when so, you can gain the so called Cholesky-decomposition of that matrix. We call a matrix $T$ to be a Cholesky-decomposition of another matrix $A$, if $A=T T^{\sim}$, where $T^{\sim}$ denotes the matrix-transposed of $T$. It is not a hard job to proof, that a Cholesky-decomposition exists if and only if when $A$ is symmetric and a positive definite.

Teachers can draw attention to the fact that when the original grid was originated by an addition using the left and the upper neighbor, then the cells of the Cholesky-decomposition will be the sum of the upper-left and the upper neighbor. The proof of this fact you can resolve from the (3a) formula [[6] p. 8] which is a special case of the Vandermonde formula.

The determinants of the main minors have got an remarkable role that also can be investigated. Let's examine grids originated from $a \equiv b \equiv c \equiv 1-$ in this case some interesting rules are, as:

- when $x \equiv z \equiv 1$ then the determinants will be of the form $(y-1)^{t}$, where $t$ is running on the triangular numbers;

- when $x \equiv 0$ and $z \equiv 1$ then the determinants will be of the form $y^{t}$, where $t$ is running on the triangular numbers;

- when $x \equiv 2$ and $\equiv 1$ then the determinants will be of the form $(y+2)^{t}$, where $t$ is running on the triangular numbers.

- The classical Pascal-triangle can be showed by the cast: $a \equiv c \equiv 1, b \equiv 0$ and $x \equiv 0, y \equiv z \equiv 1$. Or, it will perform the same triangle when using $a \equiv 1$, $b \equiv c \equiv 0$ and $x \equiv 0, y \equiv z \equiv 1$; but the table will be shifted by a column.

- If we try $a \equiv c \equiv 1, b \equiv 0$ and $x \equiv 0, y \equiv 1, z \equiv k$, we get the Stirlingnumbers of the second kind.

\section{References}

[1] E-mail by courtesy of Mr. Paul Barry.

[2] G. R. Franssens, On a number pyramid related to the binomial, Deleham, Eulerian, MacMahon and Stirling number triangles, Journal of Integer Sequences 9 (2006), Article 06.4.1.

[3] Goodman and Narayana, Lattice paths with diagonal steps., University Alberta 39 (1967). 


$$
\text { "tmcs-csabay" — 2011/5/13 — 21:42 — page } 44 \text { — \#18 }
$$

[4] http://mathworld.wolfram.com/BellTriangle.html.

[5] http://mathworld.wolfram.com/LeibnizHarmonicTriangle.html.

[6] J. Riordan, Combinatorial Identities, John Wiley \& Sons, New York, London, Sydney, 1968.

KÁROLY CSABAY and JÓZSEF DARAGÓ

UNIVERSITY OF DEBRECEN

4010 DEBRECEN, P. O. BOX 12

HUNGARY

E-mail: csabay58@gmail.com

E-mail: darago@kfrtkf.hu

(Received July, 2010) 